\title{
High Spatial Resolution X-Ray Analysis in a SEM
}

\section{Steve Chapman ${ }^{1 *}$ and Ron Rasch ${ }^{2}$}

${ }^{1}$ Protrain, 23 Foxglove Close, Buckingham MK18 1FU, United Kingdom

${ }^{2}$ Centre for Microscopy \& Microanalysis, The University of Queensland, Brisbane St Lucia, QLD 4072, Australia

*protrain@emcourses.com

\section{Introduction}

For many years energy-dispersive X-ray analysis has been carried out on scanning and transmission electron microscopes. The spatial resolution of analysis is the major difference between the two techniques, as shown in Figure 1. Working with a thin section in the transmission electron microscope (TEM), it is possible to obtain X-ray information from a region almost the same diameter as the incident electron beam. However the TEM suffers from having a sample totally surrounded by X-ray emitting materials. Careful masking of the system is required in order to ensure that $\mathrm{X}$ rays from these materials do not contribute to the X-ray spectrum from the region of interest. An analysis conducted in the scanning electron microscope (SEM) suffers from a high level of beam spread within the specimen, resulting in information from emission areas that are many micrometers in diameter and many micrometers deep. Whilst most SEM manufacturers have transmission imaging attachments, these often suffer the same problems as a TEM. They too are surrounded by materials that emit $\mathrm{X}$ rays that may interfere with the desired X-ray signal.

\section{Thin-Film X-Ray Holder}

Using a physical arrangement similar to a Faraday cup, Protrain has developed a thin-film specimen holder that allows high spatial resolution X-ray analysis in any model of SEM. The specimen sits in an aluminium holder directly above a $3 \mathrm{~mm}$ deep hole that is coated with carbon (Figure 2). The majority of electrons passing through the specimen are lost within the hole, as in a Faraday cup, and those that are backscattered are small in number because of the low backscatter coefficient of the carbon coating. The carbon coating has a secondary role in that it masks the aluminium holder reducing the aluminium systems peak in the background $[1,2]$.

There are other advantages to carrying out thin-film X-ray analysis in the SEM. In the SEM specimen chamber there is more space and therefore less possibility of interference from $\mathrm{X}$ rays generated in chamber materials. Using this technique the major contributor to artifactual $\mathrm{X}$ rays could be backscattered electrons (BSEs) hitting the bottom of the final lens, but this may be masked if a BSE detector or low-atomic-number shield is in place. When using the SEM in this mode, imaging is possible by secondary electrons (SE) and BSEs as well as through the use of X-ray maps [3]. Often BSE imaging is preferred when X-ray analysis is performed so that the electron image resolution is closer to the X-ray map resolution.

\section{Results}

Tests of the thin-film holderinaSEMwith a tungsten-hairpin source reveal useful X-ray map information at magnifications up to $100,000 \times$, where an apparent map resolution better than $100 \mathrm{~nm}$ maybediscerned. In the example shown in Figures 3 and 4 , a $\mathrm{LaB}_{6}$ source was used to demonstrate this level of X-ray map. In field-emission SEMs we have produced X-ray maps at magnifications up to $500,000 \times$. For example, thin-film analysis of polymer interfaces was carried out recently in the SEM at much higher spatial resolution than would be possible with a bulk-specimen interface [4]. Another point that may interest some scientists is that the holder also may be used in a dedicated electron probe that employs wavelength-dispersive X-ray spectrometers to produce $\mathrm{X}$-ray maps with greater elemental sensitivity.
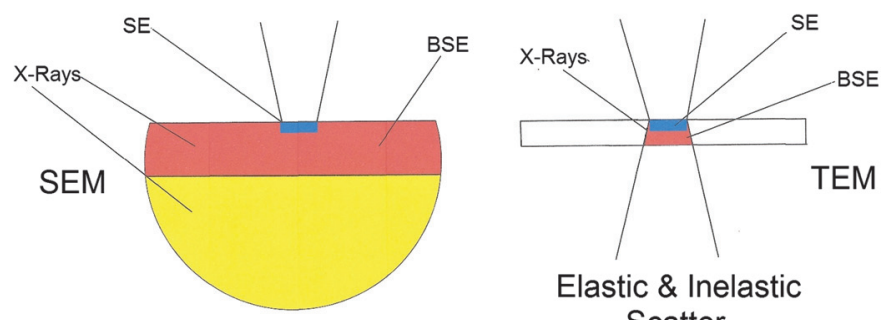

Scatter

Figure 1: Schematic comparison of the $\mathrm{X}$-ray excitation regions of a TEM thin specimen with a SEM bulk specimen.

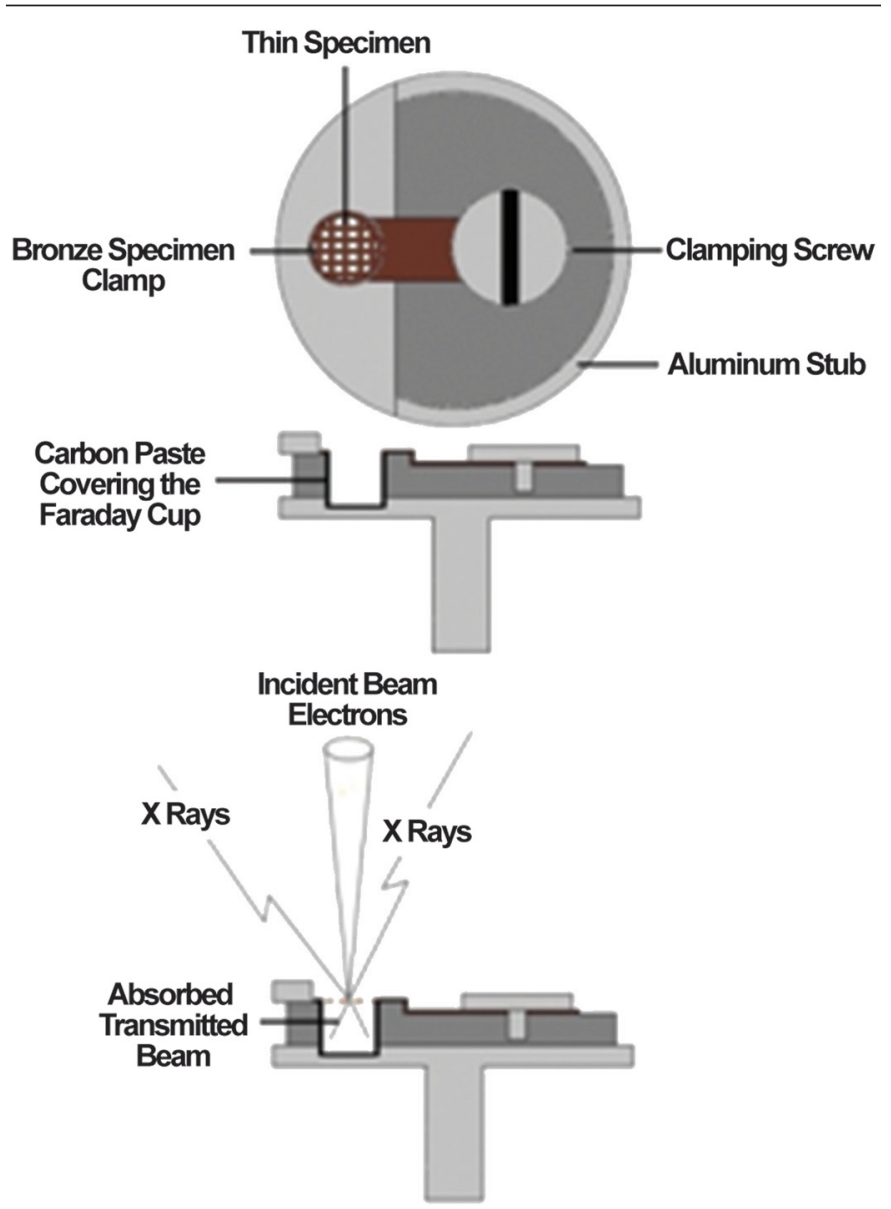

Figure 2: Schematic of the thin-film holder machined from a conventional SEM stub. 


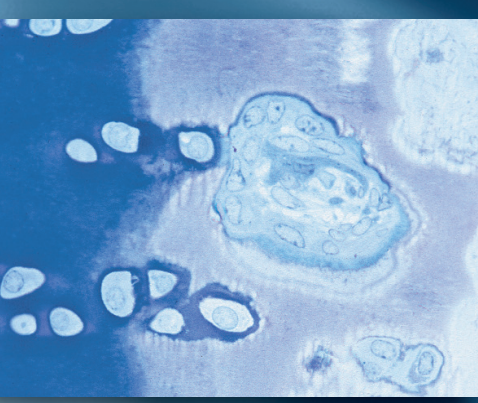

\section{DiATOME}

diamond knives

the highest quality...

the most precise sectioning... incomparable durability
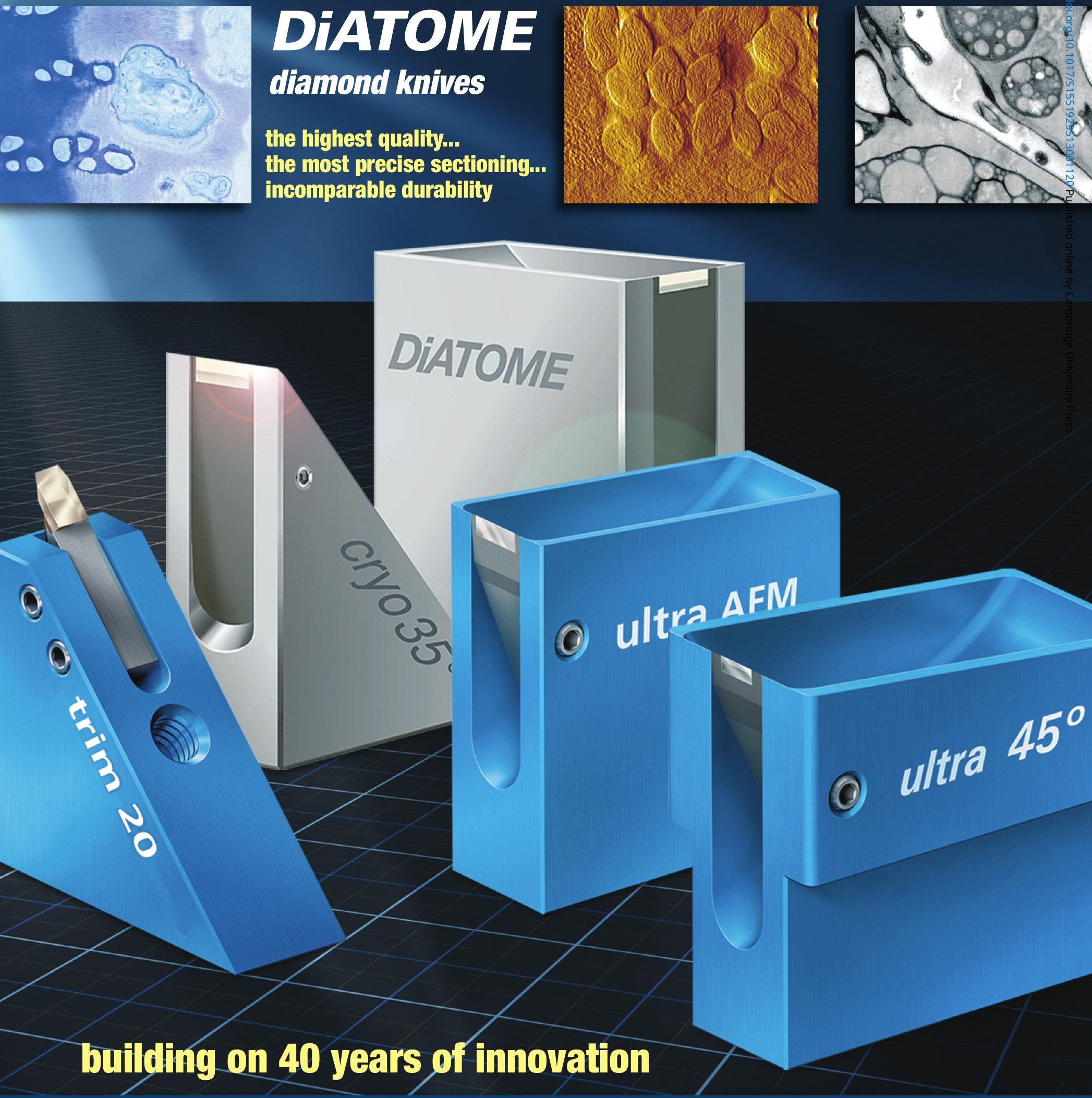

ultra $45^{\circ} \cdot$ cryo $\bullet$ histo $\bullet$ ultra $35^{\circ}$ histo jumbo • STATIC LWNE II • cryo immuno ultra sonic • ultra AFM \& Gryo AFM

NEWH.., trimtool 20 and trimtool 45

Finally, one trimming tool for all of your trimming needs, be it at room or cryo temperatures.
P.O. Box 550 • 1560 Industry Rd. • Hatfield, Pa 19440 Tel: (215) 412-8390 • Fax: (215) 412-8450 email: sgkcck@aol.com•stacie@ems-secure.com www.emsdiasum.com 


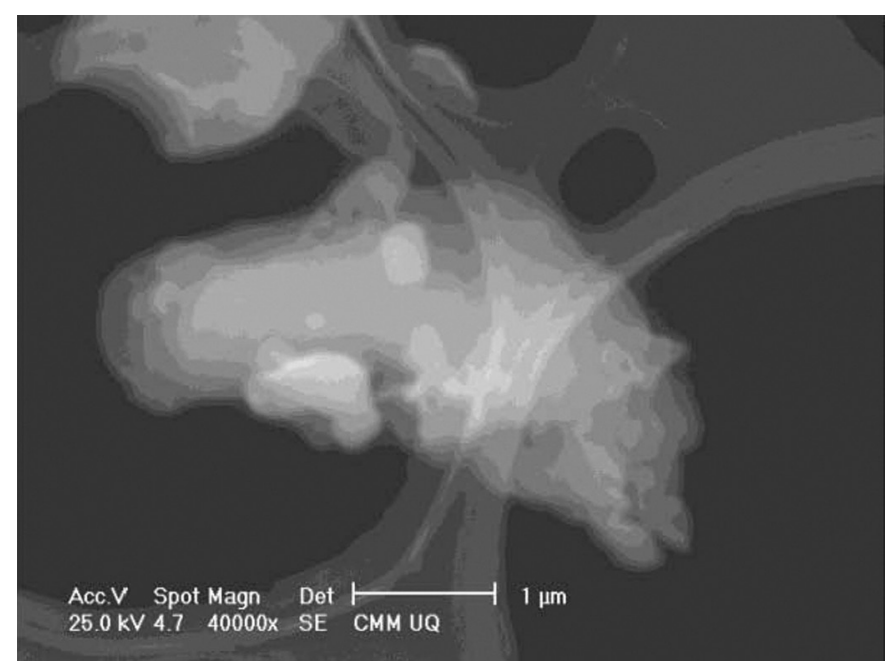

Figure 3a: Secondary electron image of a Ti-Nb-oxide particle contaminated with $\mathrm{Fe}$ acquired with the thin-film X-ray holder.

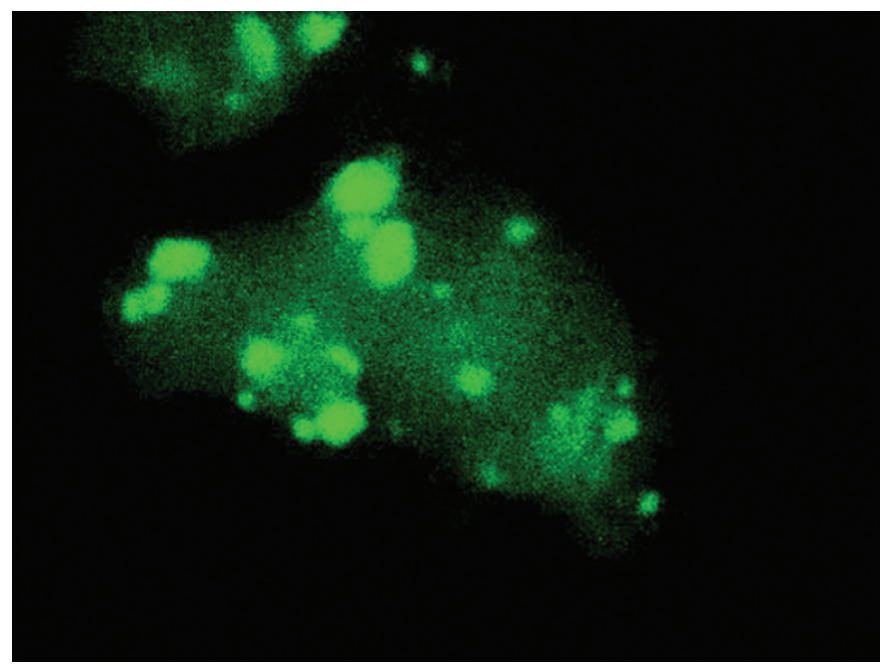

Figure 3b: Fe K $\alpha$ X-ray map acquired from the Ti-Nb-oxide particle shown in 3a.

\section{Discussion}

When carrying out thin-specimen analysis in the SEM, the analytical approach needs to be considered. Most important is that it should be realized that most SEM EDS software comes with some sort of standardless quantitative procedure. Although not as accurate as analysis using standards, this procedure is quick and in common use. It performs a matrix correction procedure (be it ZAF, $\phi(\rho z)$, or XPP [5]) to turn element X-ray peaks into a composition for the sample. In many situations, unless a thin-foil routine is provided, this SEM software will assume that the specimen is a bulk material. If applied to a thin section, the bulk matrix correction will not be appropriate and will most likely overcorrect the atomic number correction, the X-ray absorption correction, and the fluorescence correction. This leads to erroneous values for most elements. If a quantitative analysis is required, it must be treated in the same way that analysis in the TEM is performed: either (a) to ratio elemental peak intensities from the unknown and a standard before employing a modified matrix correction procedure, or (b) through the simpler Cliff-Lorimer ratio technique where $\mathrm{X}$-ray intensities from pairs of elements in the unknown specimen are ratioed to each other [6]. Others have

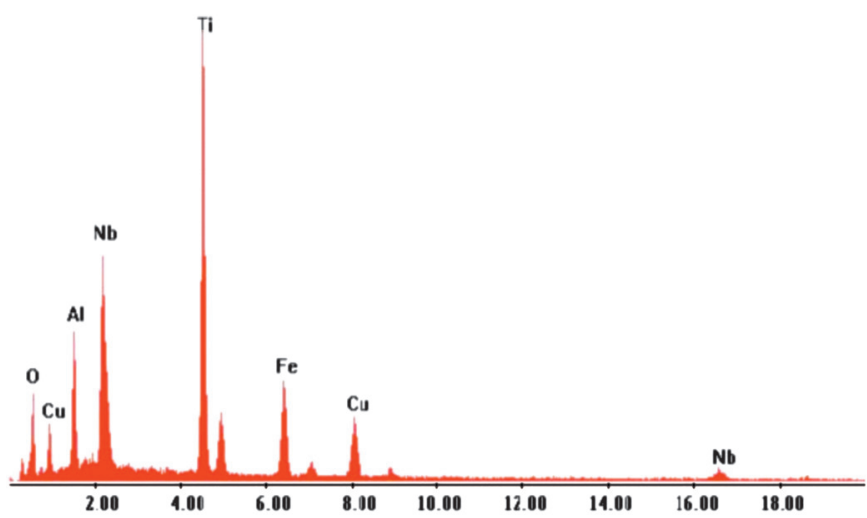

Figure 4: Global spectrum from the area examined in Figures $3 a$ and $3 b$. The horizontal scale denotes the energies of emitted $X$ rays generated with a beam energy of $25 \mathrm{keV}$. Note the low background level. (a)

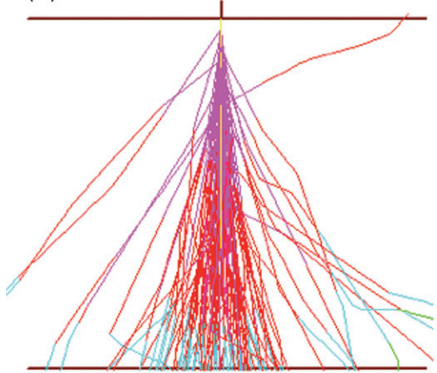

(b)

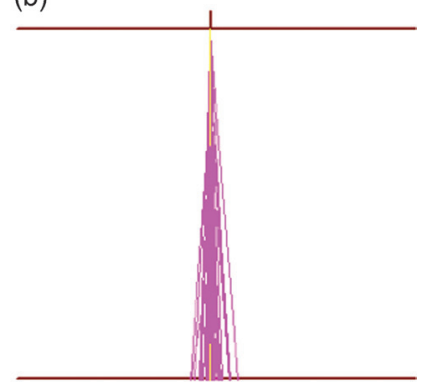

Figure 5: Monte Carlo simulations of electrons traveling through (a) $50 \mathrm{~nm}$ thick iron film at $20 \mathrm{keV}$ and (b) the same film at $200 \mathrm{keV}$.

reported using commercial TEM thin-foil standardless analysis software for thin-specimen SEM analysis and found it to produce accurate results [7]. In all cases care must be taken to ensure that analysis is not performed near a supporting grid bar.

The advantages of carrying out thin-section analytical work in the SEM are numerous. As stated previously the thin sample significantly reduces the interaction volume and therefore increases the analytical spatial resolution over normal thickspecimen SEM analysis. Thin-section analysis is traditionally done in a TEM, but we have noticed a significant increase in $\mathrm{X}$-ray count rate by moving the same specimen from the TEM to the SEM. Results will vary from lab to lab and from specimen to specimen; however it is not unusual to see an order of magnitude increase in X-ray intensity when going from TEM to SEM with the same specimen. The source of this increase in X-ray yield is twofold. First, the inner-shell ionization cross section of the specimen atoms is dependent on the accelerating voltage and, for most commonly measured X-rays, the voltages typically used in SEM analysis $(10-30 \mathrm{kV})$ produce more ionizations and therefore more X-rays than voltages used in typical TEM analysis $(100-300 \mathrm{kV})$. An overvoltage of $2-3$ is usually desirable, where overvoltage is the ratio of incident beam energy to critical ionization energy. This produces the maximum number of ionizations in most analytical situations [5,8]. Secondly, the probability of an electron scattering from a specimen atom is inversely proportional to the potential of the incident electron [5]. So electrons scatter less at the higher voltages used by TEMs, and more pass directly through the specimen. At SEM accelerating voltages, the electron beam scatters more, producing multiple 


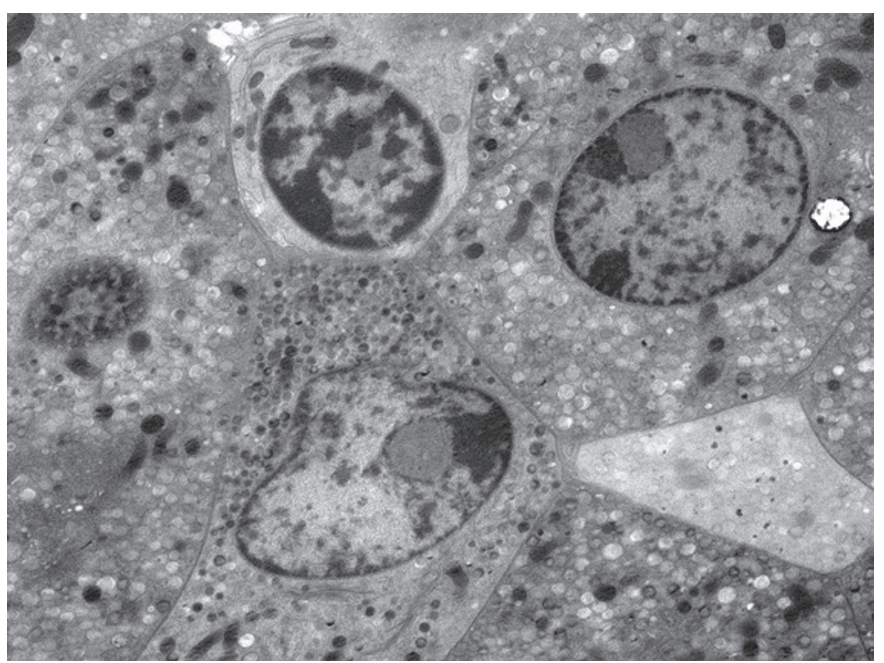

Figure 6: Stained mouse pancreatic islets of Langerhans cells imaged in the SEM in SE mode (contrast inverted). The material was high-pressure frozen, freeze substituted, fixed, and stained with uranyl acetate. The section is $60 \mathrm{~nm}$ thick.

ionizations in the specimen material, as shown by the two Monte Carlo plots (Figure 5) of electrons passing through a thin iron specimen at $20 \mathrm{kV}$ and $200 \mathrm{kV}$. These two effects together simply say that more of the beam interacts with more of the specimen at SEM voltages than at TEM voltages.

\section{Further Possibilities}

The scanning microscope may enable observation of a thin-film specimen in other advantageous modes. First, provided the specimen is mounted grid side down 100 percent of a specimen may be viewed without grid bar intervention. Secondly, SEM images of the material in inverted signal mode, particularly with stained biological material, will display an image very similar to that from a TEM in bright-field (BF) mode. Two common imaging modes in the TEM are BF mode and dark field (DF) mode, both of which are dominated by elastic scattering. In the SEM the elastically scattered signal is the BSE image, and that produces contrast similar to the TEM DF image. If a conventionally stained biological thin section is imaged in the SEM in BSE mode and then software is used to invert the contrast, the image obtained looks like a conventional biological TEM BF image.

Further, a biological thin specimen is flat and lacks topography, so the SE image contrast is now dominated by the BSE-sourced SEs of type 2 (SE II) [5]. This image also can be inverted to produce an image similar to the conventional TEM BF. Figure 6 shows a typical example is shown below of stained mouse islet sections, imaged in the SEM with SE mode; the contrast was inverted. The major advantage of this technique is that 100 percent of the specimen is available for investigation when the accelerating voltage is adjusted to minimize the grid bar signal.

\section{Conclusions}

Simply by thinking in a new dimension when examining specimens in a SEM opens up a number of new areas for investigation. Changing the style of the specimen and the way it is mounted in the microscope may offer even more information to researchers. This article describes the advantages of examining TEM thin specimens in the SEM.

\section{Acknowledgments}

The authors would like to thank David Joy for the use of his Monte Carlo modelling software, and also the facilities of the Australian Microscopy \& Microanalysis Research Facility, Centre for Microscopy and Microanalysis, The University of Queensland. Ron Rasch would like to thank Graeme Auchterlonie for his friendly support and insightful advice about electron scattering. We are both appreciative of help from Charles Lyman.

\section{References}

[1] CE Thomas and GJ Auchterlonie, "Improved Low Background Analytical SEM Holders for Thin Sections," 13th Australian Conference of Electron Microscopy, Gold Coast, February 7-14, 1994, p. 202, 7-14.

[2] A Ziegler, Microsc Microanal 13(Suppl 3) (2007) 176-77.

[3] JJ Friel and CE Lyman, Microsc Microanal 12 (2006) 2-25.

[4] L-J Vandi, R Truss, M Veidt, R Rasch, MT Heitzmann, and R Paton, J Phys Chem C 117(33) (2013) 16933-41.

[5] JI Goldstein, DE Newbury, P Echlin, DC Joy, A Romig, CE Lyman, C Fiori, and E Lifshin, Scanning Electron Microscopy and X-ray Microanalysis, $2^{\text {nd }}$ Ed., Plenum Press, New York and London, 1992, p. 69-146.

[6] DB Williams and C Barry Carter, Transmission Electron Microscopy, Plenum Press, New York and London, 1996.

[7] M Halvarsson, T Jonsson, and S Canovic, Journal of Physics: Conference Series 126 (2008) 597-620.

[8] CS Campos, MAZ Vasconcellos, JC Trincavelli, and S Segui, J Phys B-At Mol Opt 40 (2007) 3825-41.

\section{PELCO Silicon Nitride \& Silicon Dioxide Membranes}

\section{Next Generation SiN TEM Support Films}

- Robust and clean 8, 15, 50 and $200 \mathrm{~nm}$ SIN substrates

- $ø 3.0 \mathrm{~mm}$ frame

- EasyGrip ${ }^{\text {TM }}$ edges

- Free from debris

- Super flat 8, 15, and 40nm silicon dioxide substrates

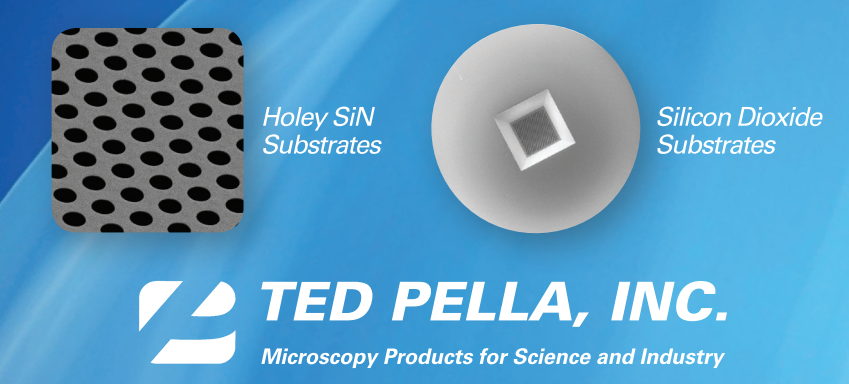

www.tedpella.com sales@tedpella.com 800.237.3526 